Revue internationale P.M.E.

Économie et gestion de la petite et moyenne entreprise

Revue

internationale

PME

\title{
Une mesure de liquidité pour les PME en croissance
}

\section{Yves Groleau}

Volume 24, numéro 3-4, 2011

URI : https://id.erudit.org/iderudit/1013662ar

DOI : https://doi.org/10.7202/1013662ar

Aller au sommaire du numéro

\section{Éditeur(s)}

Presses de l’Université du Québec

ISSN

0776-5436 (imprimé)

1918-9699 (numérique)

Découvrir la revue

Citer cet article

Groleau, Y. (2011). Une mesure de liquidité pour les PME en croissance. Revue internationale P.M.E., 24(3-4), 49-77. https://doi.org/10.7202/1013662ar

\section{Résumé de l'article}

L'objectif principal de cet article consiste à développer une mesure de liquidité adaptée à la réalité des PME en croissance (PMEC), mesure testée sur un échantillon longitudinal de 49 entreprises manufacturières québécoises présentant une information financière sur cinq années entre les périodes 1999 et 2006. Une position assez consensuelle identifie la liquidité comme étant constituée des espèces et quasi-espèces majorées de la capacité non utilisée de la marge de crédit bancaire. Par contre, les indicateurs recensés sont souvent incomplets sans donner une mesure précise du niveau de liquidité nécessaire à la capacité de s'acquitter d'un engagement financier, et ce, particulièrement chez les PMEC. Le choix d'un indicateur de liquidité moins exhaustif dans la prise de décision financière risque d'induire en erreur le gestionnaire financier dans l'estimation des liquidités futures, ce qui peut alors mettre en péril l'entreprise. Un indicateur valide constitue alors un enjeu primordial pour les propriétaires-dirigeants de ces entreprises qui désirent s'assurer un développement des affaires harmonieux sans frein ou ralentissement causé par un manque de liquidité. Une analyse de corrélation et des analyses de régression simples et multiples effectuées sur l'échantillon révèlent que la mesure développée, soit le ratio du stock de liquidité (RSL), constitue le meilleur estimateur de la liquidité future des PMEC. Les coefficients de détermination fluctuent entre $47 \%$ et $75 \%$ lorsque le RSL est estimé par le stock de liquidité et les flux de liquidité. Les caractéristiques de l'échantillon ainsi que le faible nombre d'entreprises recueillies ne permettent pas une généralisation de ces résultats à une population de PMEC. La mesure a été constituée dans un souci d'exhaustivité, et s'applique concrètement à la réalité de toute PMEC qui détient une marge de crédit bancaire.
Ce document est protégé par la loi sur le droit d'auteur. L’utilisation des services d’Érudit (y compris la reproduction) est assujettie à sa politique d'utilisation que vous pouvez consulter en ligne.

https://apropos.erudit.org/fr/usagers/politique-dutilisation/ 


\title{
Une mesure de liquidité pour les PME en croissance
}

Yves GROLEAU

Université du Québec à Trois-Rivières

MOTS CLÉS

PME en croissance (PMEC) - Ratio de stock de liquidité (RSL) Mesure de liquidité

\begin{abstract}
L'AUTEUR
YVES GROLEAU est chargé d'enseignement à I'Université du Québec à Trois-Rivières et consultant en entreprise. Ancien directeur de comptes bancaires, ses principaux champs d'intérêt touchent le développement des PME, leur financement et les pratiques de gestion. Adresse: Université du Québec à Trois-Rivières, 3351, boul. des Forges, C.P. 500, TroisRivières (Québec), Canada, G9A 5H7. Courriel: <groleauy@uqtr.ca>.
\end{abstract}

\begin{abstract}
RÉSUMÉ
L'objectif principal de cet article consiste à développer une mesure de liquidité adaptée à la réalité des PME en croissance (PMEC), mesure testée sur un échantillon longitudinal de 49 entreprises manufacturières québécoises présentant une information financière sur cinq années entre les périodes 1999 et 2006. Une position assez consensuelle identifie la liquidité comme étant constituée des espèces et quasi-espèces majorées de la capacité non utilisée de la marge de crédit bancaire. Par contre, les indicateurs recensés sont souvent incomplets sans donner une mesure précise du niveau de liquidité nécessaire à la capacité de s'acquitter d'un engagement financier, et ce, particulièrement chez les PMEC. Le choix d'un indicateur de liquidité moins exhaustif dans la prise de décision financière risque d'induire en erreur le gestionnaire financier dans l'estimation des liquidités futures, ce qui peut alors mettre en péril l'entreprise. Un indicateur valide constitue alors un enjeu primordial pour les propriétaires-dirigeants de ces entreprises qui désirent s'assurer un développement des affaires harmonieux sans frein ou ralentissement causé par un manque de liquidité. Une analyse de corrélation et des analyses de régression simples et multiples effectuées sur l'échantillon révèlent que la mesure développée, soit le ratio du stock de liquidité (RSL), constitue le meilleur estimateur de la liquidité future des PMEC. Les coefficients de détermination fluctuent entre $47 \%$ et $75 \%$ lorsque le RSL est estimé par le stock de liquidité et les flux de liquidité.
\end{abstract}


Les caractéristiques de l'échantillon ainsi que le faible nombre d'entreprises recueillies ne permettent pas une généralisation de ces résultats à une population de PMEC. La mesure a été constituée dans un souci d'exhaustivité, et s'applique concrètement à la réalité de toute PMEC qui détient une marge de crédit bancaire.

\begin{abstract}
The principal objective in this paper is to develop a liquidity measure adapted to the growth of small and medium sized enterprises (GSMEs), tested on a longitudinal panel of 49 GSMEs in the Canadian manufacturing sector over the period 1999 to 2006. Liquidity in term of financial slack is conceived as a joint condition of high cash and near-cash augmented with unused debt capacity and with the cash inflows and outflows, which are added to or subtracted from its total. As far as the liquidity component is concerned, stock and flow proxies have been variously used in the financial literacy. But theses proxies may be incomplete; they cannot be good indicators of the business' capacity to meet a payment particularly for the GSMEs. The choice of an incomplete proxy may induce the financial manager to wrong decisions. The effect can conduct the business to fail its payments and, ultimately, lead it to bankruptcy. A good proxy is a primary concern for the secure development of small or medium sized businesses. Different tests of correlation and regression analysis indicate that the LSI (Liquidity Stock Index) developed in this article represents the best measure of the future liquidity level of GSMEs. The tests for three years revealed that LSI squares between $47 \%$ and $75 \%$ when liquidity level is provided by stock and flow proxies. The characteristics of the sample and the limited number of observations do not permit a generalization for the entire population of GSMEs Canadian manufacturing businesses. The development of the LSI aims to create an exhaustive measure that can be used in practical issues by the firm's financial managers.
\end{abstract}

\title{
RESUMEN
}

El objetivo principal de este artículo consiste en el desarrollo de una medida de liquidez adaptada a la realidad de las PyMEs en crecimiento, medida probada en una muestra longitudinal de 49 empresas de manufactura en Quebec, presentando una información financiera sobre un periodo de 5 años entre 1999 y 2006. Una posición algo consensual identifica la liquidez como estando constituida de efectivo y equivalentes de efectivo aumentada de la capacidad inutilizada de la línea de crédito bancario. Sin embargo, los indicadores identificados son a menudo incompletos, sin dar una medida precisa del nivel de la liquidez necesaria para la capacidad de cumplir un compromiso financiero y esto, particularmente en las PyMEs. La elección de un indicador de liquidez menos amplio en la toma de decisiones financieras es susceptible de engañar al gerente de finanzas en la estimación de los flujos de efectivo futuros, lo que puede poner en peligro la empresa. Un indicador válido es entonces una cuestión primordial para los propietarios dirigentes de estas empresas que desean asegurar un desarrollo armonioso de negocios sin freno o disminución causado por la falta de liquidez. Un análisis de correlación y unos análisis de regresión simple y múltiples efectuados sobre la muestra revelan que la medida desarrollada, sea el indicador IEL (Indicador de Existencia en Liquidez) 
constituye la mejor estimación para la liquidez futura de las PyMEs. Los coeficientes de determinación varían entre $47 \%$ y $75 \%$ cuando el IEL se estima por la existencia en liquidez y los flujos de liquidez. Las características de la muestra y el bajo número de empresas reunidas no permiten una generalización de estos resultados para una población de PyMEs en crecimiento. La medida fue constituida con un cuidado de exhaustividad y se aplica concretamente a la realidad de toda PyME en crecimiento que tenga una línea de crédito bancario.

\section{ZUSAMMENFASSUNG}

Das Hauptziel der vorliegenden Arbeit ist die Entwicklung einer angepassten Liquiditäts-Kennzahl für wachstumsorientierte KMU. Die Kennzahl wurde anhand von Längsschnittdaten (fünf Geschäftsberichte aus den Jahren 1999 bis 2006) bei 49 Gewerbebetrieben aus Québec getestet. Allgemein wird die Liquidität als die Summe von (Quasi-) Bargeldbeständen sowie der verfügbaren Kreditlimite definiert. Die erhobenen Kennzahlen erweisen sich gerade bei wachstumsorientierten KMU als unvollständig, weil sie keine Angaben zur Fähigkeit machen, in welcher Höhe finanzielle Engagements eingegangen werden können. Entscheidungen auf Basis unvollständiger Liquiditätskennzahlen können den Fortbestand des Unternehmens gefährden. Die Entwicklung einer angepassten Kennzahl ist daher ein zentrales Thema bei KMU, um eine harmonische Geschäftsentwicklung ohne Unterbrüche durch Liquiditätsengpässe zu erreichen. Korrelations- und Regressionsanalysen ergaben, dass die entwickelte Kennzahl SLR (Stock-Liquidity-Ratio) die besten Voraussagen zum benötigten Liquiditätsbestand von wachsenden KMU ermöglichen. Die Koeffizienten der SLR liegen bei einer Gegenüberstellung von Liquiditätsbestand und -fluss zwischen $47 \%$ und $75 \%$. Aufgrund der kleinen Stichprobe ist eine Generalisierung auf sämtliche Wachstumsbetriebe unter den KMU nicht angebracht, eine Anwendung für Unternehmen mit einer Kreditlimite scheint allerdings angebracht.

\section{Introduction}

L'importance de la liquidité et des flux monétaires dans la gestion financière des PME est soulignée par McMahon (2004), qui l'associe à une question de survie de l'entreprise. Il rapporte de l'étude de Welsh et White (1981) que les PME peuvent survivre assez longtemps sans bénéfices, mais s'approchent de la faillite dès qu'elles doivent manquer un paiement important. Dans un tel sens, la liquidité constitue la somme disponible pour respecter les engagements financiers (Welsh et White, 1978), pour faire un chèque. Une définition assez consensuelle (Emery, 1984; Lemke, 1970; De la Bruslerie, 1999; McMahon, 2004) la décrit comme étant constituée du stock et des flux de liquidité. Détenir une liquidité suffisante est une condition essentielle à la survie des PME, mais touche particulièrement les PMEC, où les besoins de la croissance rendent le stock de liquidité plus volatil. 
Caractériser les PME par la croissance aide à mieux les comprendre, elles qui forment un ensemble hétérogène d'entreprises. Les PMEC constituent la pierre angulaire du développement économique. Julien (2002) mentionne que les PMEC forment de $2 \%$ à $10 \%$ des entreprises pérennes, mais créent de $40 \%$ à $60 \%$ des nouveaux emplois. L'étude des entreprises en forte croissance intéresse les chercheurs et les pouvoirs publics, autant pour l'importance de leur impact sur le développement économique que pour leurs particularités. Parmi celles-ci, un intérêt marqué est porté à l'analyse de facteurs qui influencent leur réussite, leur pérennité et leur risque. Ces entreprises évoluent dans un milieu turbulent où les liquidités sont instables et où les besoins doivent être rapidement comblés, afin d'assurer un développement harmonieux. Ces besoins, traduits en termes financiers, sont satisfaits par les ressources actuelles, celles générées par les activités ainsi que par les financements obtenus, tout en laissant idéalement un surplus de liquidité. Les PMEC qui n'arrivent pas à couvrir leurs besoins financiers risquent le ralentissement de leur croissance, pouvant aller jusqu'à la cessation de leurs activités (Binks et Ennew, 1996; Churchill et Lewis, 1983; Carpenter et Petersen; 2002, Welsh et White, 1981; McMahon, 2004). Des précisions sur la dynamique de la croissance ainsi que sur les ressources financières, physiques et humaines disponibles et nécessaires au développement de la PME peuvent alors contribuer à mieux cerner les problèmes anticipés de la PMEC et y proposer d'éventuelles solutions.

Il est montré par le rapport américain sur la petite entreprise (United States Government Printing Office, 2007) et divers autres auteurs (Berger et Udell, 1995, 1998), que les PME se tournent naturellement vers le financement bancaire pour combler leurs besoins à court terme, le pourcentage d'entreprises financées augmentant avec la taille. Cette relation d'affaires naturelle soulève un problème d'asymétrie d'information (Jensen et Meckling, 1976) entre la banque et l'entreprise, ce problème étant exacerbé chez les PME, qui ont une opacité informationnelle si on les compare aux plus grandes entreprises (Berger et Udell, 1998). Pour se prémunir contre ce problème d'asymétrie d'information, les banques se dotent de mécanismes de suivi, de limitation et de garanties (Berger et Udell, 1995). Cet effet «protecteur» oblige les PMEC à partager le risque d'affaires avec ce partenaire en injectant une mise de fonds, réductrice de liquidité, pour financer leurs besoins financiers. Le financement bancaire constitue alors une solution partielle, mais non complète aux besoins de la croissance. Peut-on penser que ces mécanismes compensateurs peuvent provoquer des contraintes financières? 
Myers et Majluf (1984) soulignent les besoins supplémentaires de liquidité requis par la croissance, tout comme le risque de manque de liquidité pour l'acceptation de projets à VAN positive. D'autres auteurs (Hyytinen et Toivanen, 2005; McMahon, 2001; Carpenter et Petersen, 2002) montrent que les contraintes financières peuvent restreindre la croissance et l'innovation chez les PME. De plus, les résultats de l'étude de St-Pierre, Beaudoin et Desmarais (2002) indiquent que la croissance réduit le taux d'acceptation des prêts bancaires à court terme. Si la croissance accroît la pression sur les besoins de liquidité, et que les partenaires financiers ne parviennent pas à l'alléger, alors les PMEC doivent se tourner vers d'autres sources de financement ou des méthodes génératrices de liquidité pour répondre à leurs besoins supplémentaires, notamment l'autofinancement.

À ce sujet, un courant émergeant de la littérature, le «bootstrap financing» (Winborg et Landsrom, 2001; Van Auken et Neeley, 1996), identifie des modes de solutions préconisés par les entreprises qui veulent éviter le recours au financement externe. Les chercheurs valident empiriquement l'utilisation de diverses pratiques de gestion qui favorisent l'économie de liquidité. Bien que les bonnes pratiques de gestion servent à générer davantage de liquidité, notamment chez la PMEC, elles ne guident pas le propriétaire-dirigeant quant au niveau de liquidité nécessaire au soutien du développement de l'entreprise. Il reste que, pour le propriétaire-dirigeant, il est impératif de déterminer l'ampleur du besoin financier.

La problématique managériale que vivent les dirigeants de la PMEC touche deux volets, soit la détermination du besoin financier à combler, tout comme le niveau de connaissances, par les parties prenantes, de l'étendue des besoins de liquidité requis par la croissance. Dans cette optique, l'objectif de cet article vise à mieux comprendre les besoins des PMEC par le développement d'une mesure exhaustive de liquidité, permettant de quantifier avec justesse les besoins et les sources de financement. Une mesure moins exhaustive, qui omet des constituantes de la liquidité, risque de mal tenir compte des besoins de liquidité de la PMEC. Bien que cette recherche soit exploratoire, elle vise à clarifier le choix d'un indicateur valide de liquidité chez les PMEC.

Dans les prochaines sections seront présentés le contexte théorique, où sera développée la mesure, puis la méthodologie de la recherche et les résultats obtenus. Ceux-ci permettront de mieux comprendre la motivation à développer une mesure exhaustive, feront ressortir la pertinence d'inclure une mesure où le stock et le flux de liquidité contribuent à la détermination de la liquidité et justifieront le choix d'une mesure fondée sur la liquidité future. 


\section{Cadre théorique et développement du modèle}

Le développement d'une mesure de liquidité pour les PMEC nécessite d'apporter des précisions sur le concept de liquidité et ses indicateurs. Une présentation du contexte dans lequel évoluent les PMEC justifie les choix préalables au développement du modèle. La prochaine section précise le lien entre les préférences du propriétaire-dirigeant et les choix de financement adoptés par l'entreprise.

\subsection{Les objectifs des propriétaires-dirigeants et les choix de financement}

Dans la mesure où les préférences du propriétaire-dirigeant peuvent avoir une influence sur les choix financiers effectués dans l'entreprise, il est important de comprendre ces préférences et d'établir leur impact sur les choix qu'il fera.

Naffziger, Hornsby et Kuratko (1994) indiquent que les objectifs personnels du propriétaire-dirigeant d'une PME se confondent à ceux de son entreprise. LeCornu, McMahon, Forsaith et Stanger (1996) citent les études de Cooley et Edwards (1983), ainsi que celles de Ray et Hutchinson (1983), qui expliquent que les objectifs du propriétaire-dirigeant de PME ne sont pas uniquement financiers, mais plus nombreux et complexes. Walker et Brown (2004) constatent que des critères financiers et non financiers motivent les décisions des propriétaires-dirigeants, les critères non financiers dominant les critères financiers. LeCornu et al. (1996) proposent une fonction d'utilité pour les propriétaires-dirigeants constituée notamment de rendements financiers et non financiers.

Si la satisfaction du propriétaire-dirigeant est principalement liée à des rendements non financiers, et que ces derniers touchent des éléments tels que le désir d'indépendance, le désir de préservation du style de vie personnelle, la contribution à la société et le désir d'être respecté (LeCornu et al.,1996), alors les choix de financement dans l'entreprise peuvent être dictés par ces préférences.

À ce sujet, diverses théories contribuent à expliquer les choix de financement qui sont effectués par les propriétaires-dirigeants d'entreprise. La théorie du cycle de vie stipule que les choix de financement peuvent dépendre du positionnement de l'entreprise dans son cycle de vie. Les PMEC recourent plus naturellement à l'endettement pour le financement de la croissance. Selon la théorie du choix managérial, le dirigeant orienterait la structure de financement en fonction de ses désirs et préférences financières (St-Pierre, 1999). La théorie du compromis pose que le dirigeant hésite entre les avantages fiscaux à l'endettement et les risques de 
faillite qu'il comporte. Ces choix sont dictés notamment par un souci de maximisation de la valeur de la société induit par une minimisation du coût du capital. De ces théories émerge un constat confirmant l'influence du propriétaire-dirigeant dans les choix de financement de la PMEC. La mise en parallèle de ces théories avec celle de l'agence explique en partie la motivation que peuvent avoir les dirigeants des PMEC à privilégier l'autofinancement et les pratiques d'affaires génératrices de liquidité. Avec une insuffisance de financement bancaire (ou provenant des intermédiaires financiers), un manque éventuel de liquidité devrait alors être comblé par du financement par fonds propres.

À ce sujet, la théorie de l'ordre hiérarchique suggère un ordre adopté par les propriétaires-dirigeants dans leurs choix de financement. Divers auteurs ont constaté un ordre hiérarchique que peuvent se donner les PME dans leurs choix de financement, notamment Bird et Jüttner (1975), Myers (1984), Holmes et Kent (1991) et Zoppa et McMahon (2002). Quoique les études divergent entre elles, il en ressort des éléments importants:

- Les PME préfèrent le financement par fonds internes.

- Lorsque nécessaire, elles optent tout d'abord pour un financement externe sous forme d'endettement.

- Elles visent ensuite le recours aux fonds propres externes, uniquement si les propriétaires-dirigeants sont disposés à le faire (Holmes et Kent, 1991; Howorth, 2001).

Cette séquence de préférences suggère une séparation des divers modes de financement dans les décisions ou modèles visant à déterminer les fonds nécessaires au financement de la croissance. C'est dans ce cadre conceptuel particulier que sera développée et opérationnalisée la mesure de liquidité utile aux PMEC.

Le développement de la mesure suggère de s'intéresser à ses constituantes, qui sont liées aux modes de présentation de l'information financière. La prochaine sous-section décrit les constituantes du stock et des flux de liquidité.

\subsection{La liquidité}

La liquidité constitue le réservoir financier de l'entreprise. La liquidité attendue $\left(\mathrm{L}_{t}\right)$ peut être décomposée en deux parties, les éléments de stock de liquidité de la période précédente $\left(\mathrm{L}_{t-1}\right)$ et les flux de liquidité de la période, qui, eux, se répartissent selon les recettes $\left(\mathrm{REC}_{t}\right)$ et les déboursés $\left(\mathrm{DÉB}_{t}\right)$ de la période (équation 1). 
$\mathrm{L}_{t}=\mathrm{L}_{t-1}+\mathrm{REC}_{t}-\mathrm{DÉ}_{t}$

Équation 1

\subsubsection{Le stock de liquidité}

À tout moment du temps, le stock de liquidité (équation 2) se compose des montants d'espèces $(\mathrm{E})$ et de quasi-espèces $(\mathrm{QE})$, moins le découvert bancaire (DB), s'il existe, et majorés du solde inutilisé de la marge de crédit bancaire (MCD). Les trois premiers termes sont disponibles dans le bilan et constituent le solde net d'encaisse réelle. Le dernier terme n'est pas disponible dans les états financiers et s'ajoute aux trois premiers termes pour indiquer la liquidité potentielle de l'entreprise, somme utile pour l'évaluation du montant disponible pour faire un chèque. Il se calcule (équation 3 ) en soustrayant le montant utilisé périodiquement (MCU) du montant de crédit autorisé lors de cette période (MCA). Quant à cette dernière somme (équation 4), elle est limitée au moindre des deux montants suivants: le montant négocié au contrat de prêt (MCC) ou la valeur calculée des biens offerts en garantie. Ce dernier calcul constitue la moyenne pondérée des actifs donnés en garantie (A), chacun de ceux-ci étant pondéré par la proportion maximale admise $(p)$ par la banque en couverture des sommes allouées. De ce total sont retranchés les liens prioritaires (PR) détenus sur les actifs. Ce calcul résulte directement du mécanisme restrictif de limitation instauré par la banque en réaction au problème d'asymétrie d'information. Il est effectué périodiquement (mensuellement) par la banque. Il est alors possible que le résultat limite le montant disponible périodiquement lorsque la valeur pondérée des actifs en garantie ne couvre pas celle inscrite au contrat. Dans ces situations, la liquidité disponible s'en trouve réduite.

$\mathrm{L}=\mathrm{E}+\mathrm{QE}-\mathrm{DB}+\mathrm{MCD}$

Équation 2

$\mathrm{MCD}=\mathrm{MCA}-\mathrm{MCU}$

Équation 3

$\mathrm{MCA}=\min \left[\mathrm{MCC}, \sum_{i=1}^{n}\left(\mathrm{p}_{i} \mathrm{~A}_{i}-\mathrm{PR}_{i}\right)\right]$

Équation 4

Cette mesure du stock de liquidité s'applique à toute entreprise qui doit offrir des garanties à une institution financière pour justifier l'utilisation d'une marge de crédit. Dans le cas où ce mode de contrôle est inexistant, alors l'équation 4 correspond au montant établi au contrat de crédit. 
Les flux de liquidité sont constitués de la variation du stock de liquidité entre deux périodes. Nous allons les décrire en détail.

\subsubsection{Les flux de liquidité}

Comme le stock de liquidité ne comprend pas uniquement les espèces et les quasi-espèces, mais également le solde inutilisé du crédit bancaire, les flux de liquidité se décomposent en deux parties:

- La variation des flux monétaires de la période (décrite à l'état des flux de trésorerie) qui affecte la valeur des espèces, des quasiespèces, du découvert bancaire et du solde de la marge de crédit.

- La variation du montant de marge de crédit disponible qui touche le solde inutilisé du crédit bancaire.

La seconde composante est liée au fait que la liquidité potentielle dépend de la variation du montant contractuel de marge de crédit négocié avec la banque et des valeurs offertes en garantie. Ainsi, une entreprise qui majore sa marge de crédit de $500000 \$$ à $1000000 \$$ voit sa liquidité potentielle majorée d'autant. La renégociation de la marge de crédit entraîne cependant un coût supplémentaire (coût de renégociation, frais de dossier, etc.) visant notamment à réduire l'asymétrie d'information entre l'entreprise et la banque (Binks et Ennew, 1996).

La première composante est présentée à l'état des flux de trésorerie, état financier qui fait ressortir les changements dans les activités d'exploitation, d'investissement et de financement. La présentation d'une mesure de liquidité qui provient du bilan ou qui présente uniquement une portion de l'ensemble des flux monétaires risque invariablement d'être invalide. Le souci d'exhaustivité dans la dérivation de la mesure émane de cette prérogative et se justifie par un accès actuel plus facile aux outils de calcul requis par les dirigeants de l'entreprise. Dans un tel contexte, il est possible que la mesure calculée soit plus complexe pour un utilisateur ou une partie prenante externe, l'objectif étant de résoudre une problématique managériale.

Les flux de liquidité peuvent être remaniés sans incidence sur la valeur calculée. Un remaniement de la forme de présentation des flux de liquidité aura deux fins principales:

- Séparer les recettes des déboursés.

- Faire ressortir les options offertes à l'entreprise dans ses modes de financement, comme suggéré par les théories financières, dont celle de l'ordre hiérarchique. 
La présentation proposée restructure l'état des flux de trésorerie selon la méthode indirecte. Cette dernière est préférée à la méthode directe, qui décrit les flux monétaires opérationnels en entrées et sorties de fonds au lieu de les présenter en fait de variations du bénéfice net. Selon la méthode indirecte et la théorie de l'ordre hiérarchique ${ }^{1}$, on ordonne les recettes comme suit:

- Les fonds autogénérés, soit le bénéfice net $(\mathrm{BN})$ plus les charges ne donnant pas lieu à des sorties de fonds (AM). La plupart du temps, ces flux monétaires sont positifs, mais ils peuvent également prendre une valeur négative.

- La variation de la marge de crédit bancaire (MCD). Cet élément n'est pas inclus dans les flux de trésorerie, mais constitue une part importante des flux de la liquidité, touchant ici la variation de la capacité non utilisée du crédit bancaire.

- Les nouveaux fonds propres amicaux (NFPA), qui constituent les ajouts au «dû aux actionnaires» ou au capital-actions, à condition de pouvoir les dissocier des ajouts provenant d'investisseurs externes.

- Les nouveaux fonds propres externes (NFPE), qui constituent les injections en capital-actions provenant d'investisseurs externes à l'entreprise.

Voici les éléments constitutifs des déboursés:

- Les versements de capital exigibles dans l'année $\left(\mathrm{PCTDLT}_{\mathrm{t}-1}\right)$. Ces déboursés sont inscrits en premier, car ils constituent des engagements financiers déjà contractés.

- Le versement de dividendes (DIVID). Puisque ce versement diminue les bénéfices de l'exercice transférés aux bénéfices non répartis, on le retranche des déboursés.

- Le besoin en fonds de roulement (FDR). Il est constitué de la variation des postes de fonds de roulement, soit les comptes clients (CC), plus les stocks (S), plus les charges payées d'avance (CPA), moins les comptes fournisseurs $(\mathrm{CF})$, moins les frais courus à payer $(\mathrm{FCP})$. Aux fins de simplification, la variation des charges payées d'avance et des frais courus à payer est intégrée à celle des comptes fournisseurs.

1. Remarquons que le choix de la théorie de l'ordre hiérarchique ne sert ici qu'à l'organisation de l'information financière. 
- Les nouvelles immobilisations (NIMM). Elles peuvent être regroupées avec leur financement sous forme d'emprunts à long terme (NDLT). Aux fins de simplification, on peut soustraire de ce besoin les dispositions en actifs immobilisés (DA) et ajouter les sommes obtenues sous forme de refinancement. L'expression devient alors NIMM -NDLT ou NIMM (1 -NDLT/NIMM).

La prochaine étape consiste à décomposer le stock de liquidité attendu par la portion du stock de liquidité de la période précédente, majorée des flux de liquidité de la période courante. Cette décomposition rappelle l'autocorrélation entre les montants de stock de deux exercices consécutifs et fait ressortir l'impact de la variation des flux monétaires sur la valeur du stock résultant.

\subsubsection{Relation entre stock et flux de liquidité}

Il est possible de regrouper l'ensemble de cette information financière exhaustive en décomposant l'équation 1.

$\mathrm{L}_{t}=\mathrm{L}_{t-1}+\mathrm{REC}_{t}-\mathrm{DÉB}_{t}=\mathrm{L}_{t-1}+\left(\mathrm{BN}_{t}+\mathrm{AM}_{t}\right)+\Delta \mathrm{MCD}_{t}+\mathrm{NFPA}_{t}+\mathrm{NFPE}_{t}$

$-\mathrm{PCTDLT}_{t-1}-\mathrm{FDR}_{t}-\mathrm{DIVID}_{t}-\mathrm{NIMM}_{t}\left(1-\frac{\mathrm{NDLT}_{t}}{\mathrm{NIMM}_{t}}\right)$

Équation 5

L'équation 5 remplit les conditions d'exhaustivité recherchées et permet donc de créer une mesure non biaisée qui exprime la liquidité des PMEC. C'est avec cette base théorique que se constitue la mesure proposée: on y fera également référence aux fins de comparaison avec les autres mesures recensées.

Comme le développement du modèle s'applique dans un contexte de PMEC, nous allons préciser le concept de croissance.

\subsection{La croissance}

La croissance peut être exprimée selon diverses mesures et par divers estimateurs (Delmar, Davidsson et Gartner, 2003). Trois mesures ont été répertoriées dans la littérature: la croissance des ventes, du nombre d'employés et de l'actif. Comme les résultats (ventes) proviennent d'une contribution du capital (actif) et du travail (nombre d'employés), et que la plupart des études financières (Becchetti et Trovato, 2002; Birley et Westhead, 1990; Hyytinen et Toivanen, 2005; McMahon, 2001) choisissent la croissance des ventes comme mesure, nous avons opté pour la croissance des ventes comme 
concept. Rappelons que la variation des flux monétaires touche l'état des flux de trésorerie qui s'exprime en fonction du bénéfice net, résultante de la différence entre les produits (ventes) et les charges.

\subsection{Le cadre conceptuel et la question de recherche}

FIGURE 1

Cadre conceptuel de la recherche

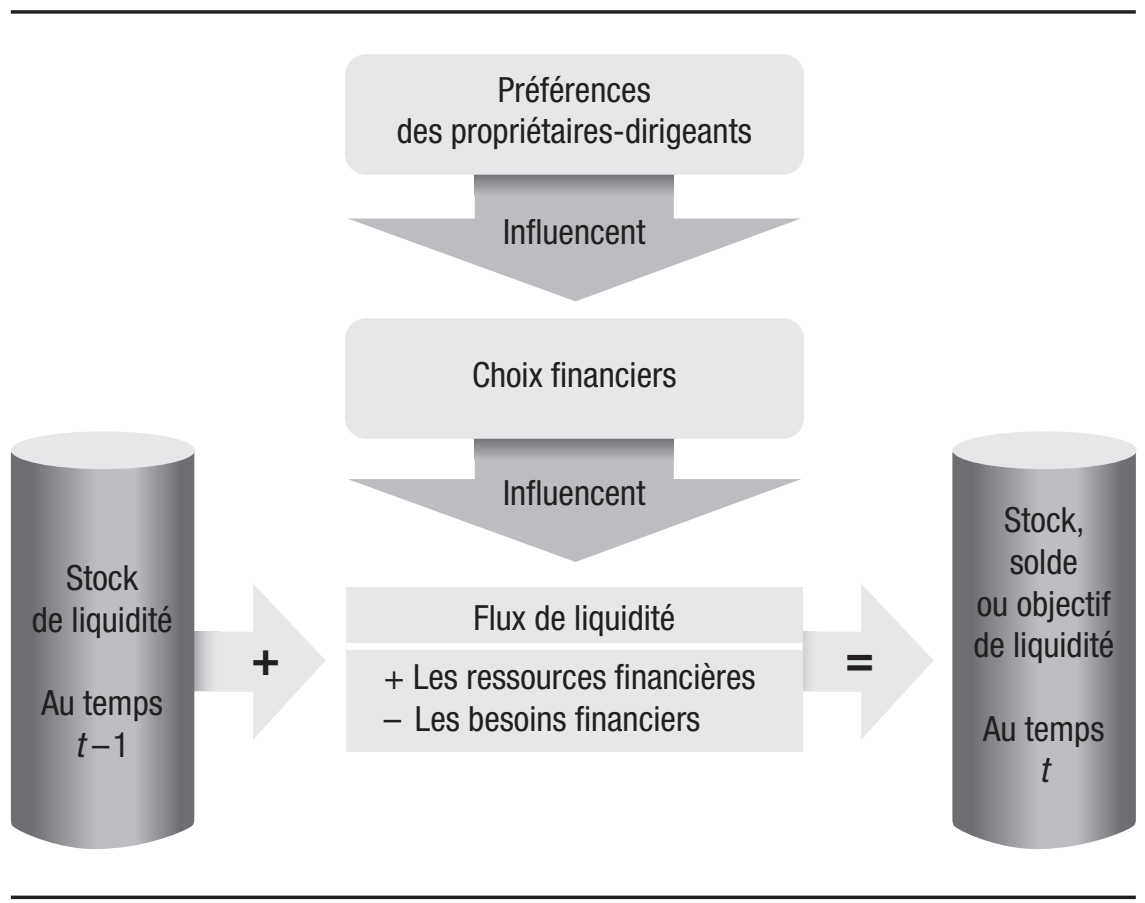

Le contexte théorique décrit la liquidité comme étant constituée du stock de liquidité de la période précédente et des flux de liquidité de la période actuelle. Ce stock de liquidité constitue davantage un paramètre qu'une variable, car il est connu d'avance. Les flux de liquidité sont variables et soumis aux préférences des propriétaires-dirigeants. Les décisions de ces derniers influencent les choix financiers (niveau des ventes, besoins et sources de financement), qui agissent sur les besoins et sources de financement. La figure 1 présente ce cadre conceptuel. Ces facteurs de détermination de la liquidité suggèrent la question de recherche suivante: 


\subsubsection{Quelle mesure de liquidité convient aux PMEC?}

La réponse à cette question de recherche est liée à l'objectif de recherche qui consiste à quantifier avec précision les besoins et sources de financement. Un critère d'exhaustivité dans le choix de la mesure est donc recherché. La mesure choisie doit inclure le stock et les flux de liquidité. Elle vise à éclairer le gestionnaire financier sur la sensibilité des éléments constitutifs du stock et des flux de liquidité, qui dépendent du niveau des ventes et des choix corporatifs. La décomposition possible de la mesure en différentes parties mettrait en évidence le lien entre le niveau de la liquidité et les modes de financement disponibles, comme le suggèrent les théories financières. La sensibilité sur le plan des ventes justifie alors le choix du concept de croissance.

La prochaine section propose donc une constitution de la mesure.

\subsection{Constitution de la mesure}

Afin de respecter le souci d'exhaustivité, condition essentielle pour éviter des biais de mesure, nous avons créé la mesure RSL en fonction de la dérivation proposée à la sous-section précédente. De cette manière, aucun élément constitutif de la liquidité ne sera omis. Afin de rendre la mesure comparable d'une entreprise à l'autre, nous avons divisé le tout par le niveau des ventes $(\mathrm{V})$.

$$
\begin{aligned}
& \mathrm{RSL}_{t}=\frac{\mathrm{L}_{t}}{\mathrm{~V}_{t}}=\frac{\mathrm{E}_{t}+\mathrm{QE}_{t}-\mathrm{DB}_{t}+\mathrm{MCD}_{t}}{\mathrm{~V}_{t}} \\
& =\frac{\mathrm{L}_{t-1}-\mathrm{FDR}_{t}-\mathrm{DIVID}_{t}-\mathrm{PCTDLT}_{t-1}-\mathrm{NIMM}_{t}\left(1 \frac{\mathrm{NDLT}_{t}}{\mathrm{NIMM}_{t}}\right)+\mathrm{MCD}_{t}+\mathrm{NFPA}_{t}+\mathrm{NFPE}_{t}}{\mathrm{~V}_{t}}
\end{aligned}
$$

Équation 6

Cette mesure (équation 6) possède plusieurs caractéristiques intéressantes:

- Elle permet d'obtenir le montant exact disponible pour faire un chèque si on connaît la valeur du chiffre d'affaires.

- Elle s'exprime en pourcentage du chiffre d'affaires, ce qui est facile à saisir pour le propriétaire-dirigeant .

- Elle décrit à la fois le stock et les flux de liquidité.

- Elle se décompose aisément, ce qui facilite les analyses de sensibilité sur lesquelles reposent les choix corporatifs. 
- Elle sert à évaluer la liquidité future.

- Elle est exhaustive et ne comporte donc pas de biais.

La prochaine section présente diverses mesures émanant de la littérature, mesures qui seront comparées avec le ratio RSL afin de déterminer le meilleur indicateur du stock de liquidité. Rappelons que la liquidité future représente le stock de liquidité de la période actuelle majoré des flux de liquidité futurs.

\subsection{Autres mesures de liquidité et leurs limites}

Les mesures relevées dans la littérature sont divisées en trois catégories, celles émanant du bilan, celles émanant du cycle de conversion de l'encaisse et celles à base de flux monétaires. Elles sont présentées et commentées en fonction de la mesure de liquidité recherchée.

\subsubsection{Mesures émanant du bilan}

Les ratios dérivés du bilan figurent parmi les plus anciens et les plus utilisés de tous les ratios. Trois mesures ont été retenues, soit:

1. Le ratio de fonds de roulement: $\mathrm{RFDR}=\frac{\text { Actif à court terme }}{\text { Passif à court terme }}$

Équation 7

2. Le ratio de liquidité immédiate: $\mathrm{RLI}=\frac{\text { Actif à court terme }- \text { Stocks }}{\text { Passif à court terme }}$

Équation 8

3. Le solde de liquidité nette ${ }^{2}$ :

$\mathrm{SLN}=\mathrm{CP}-\mathrm{BFR}=(\mathrm{AV}+\mathrm{DLT}-\mathrm{IMM})=\mathrm{E}+\mathrm{QE}-\mathrm{BP}-\mathrm{PCTDLT}-\mathrm{MCU}$

Équation 9

2. Le SLN s'obtient en soustrayant le besoin du fonds de roulement (BFR) du capital permanent (CP). Le capital permanent est constitué de l'avoir (AV) et de la dette à long terme (DLT), desquels on retranche la valeur des actifs à long terme (AMM). Le besoin de fonds de roulement est obtenu en additionnant les comptes clients, les stocks et les charges payées d'avance qui sont réduites des comptes fournisseurs et des frais courus à payer. Il est donc constitué des espèces (E) et quasi-espèces $(\mathrm{QE})$, desquelles on retranche le solde de la marge de crédit $(\mathrm{MCU})$, des billets à payer $(\mathrm{BP})$ et la portion de la dette à long terme venant à échéance lors de l'exercice (PCTDLT). 
Le ratio du fonds de roulement est bien connu dans la littérature financière. Sa simplicité de calcul ainsi que sa popularité en font un outil très prisé en analyse financière. Son interprétation indique dans quelle mesure les actifs à court terme couvrent les passifs à court terme en cas de liquidation; en ce sens, il mesure la solvabilité. Le ratio de liquidité immédiate corrige le ratio de fonds de roulement pour l'évaluation de la liquidité au numérateur en retranchant le stock et s'interprète de la même manière.

Conceptuellement, ces ratios ne sont pas acceptables pour la constitution d'une mesure adaptée aux PMEC du fait qu'ils sont incomplets, ne donnent pas d'interprétation de la position future de la liquidité et n'indiquent pas au propriétaire-dirigeant le montant disponible pour faire un chèque. Un ratio incomplet risque d'induire le dirigeant en erreur dans son interprétation de la liquidité disponible. De plus, ce sont des ratios de stock de liquidité; ils ne sont pas exprimés en fonction des ventes. Bien que le ratio de liquidité immédiate corrige en partie le problème de la constituante de liquidité du ratio de fonds de roulement, les éléments du numérateur demeurent à la fois des besoins et des sources de liquidité.

Le SLN propose une décomposition du fonds de roulement qui aide à vérifier si la variation dans les liquidités est attribuable au cycle d'opération ou aux fluctuations dans le capital permanent de l'entreprise. Ce raisonnement suppose que la dégradation du fonds de roulement peut être provoquée par d'autres éléments que les composantes du cycle d'exploitation. Ce ratio présente les mêmes limites que les deux précédents, à savoir qu'il ne procure pas d'information sur les flux monétaires futurs, sur la sensibilité eu égard aux ventes ainsi que sur le montant disponible pour s'acquitter d'un engagement financier.

La popularité et les limites des ratios émanant du bilan, largement diffusées dans la littérature financière, ont incité certains auteurs, dont De la Bruslerie (1999) et Richards et Laughlin (1980), à corriger une partie de leurs déficiences en liant les postes du bilan à ceux des résultats.

\subsubsection{Mesures du cycle de conversion de l'encaisse}

Les mesures du cycle de conversion de l'encaisse servent à évaluer le délai de conversion des activités de l'entreprise en encaisse. Deux mesures ont été répertoriées:

$\begin{aligned} & \mathrm{CCE}=\mathrm{DDCC}+\mathrm{DDS}-\mathrm{DPCF} \\ & \text { 1. Le cycle de conversion de l'encaisse : } \mathrm{CCE}=\frac{\mathrm{CC}}{\mathrm{V} / 365}+\frac{\mathrm{S}}{\mathrm{CMV} / 365}-\frac{\mathrm{CF}}{\mathrm{CMV} / 365}\end{aligned}$ 
a. Le cycle de conversion de l'encaisse ajusté:

$$
\begin{array}{ll}
\mathrm{CCEA} & =a \mathrm{DDCC}+b \mathrm{DDS}-c \mathrm{DPCF} \\
\text { ajusté }: & \mathrm{CCEA}=a \frac{\mathrm{CC}}{\mathrm{V} / 365}+b \frac{\mathrm{S}}{\mathrm{CMV} / 365}-c \frac{\mathrm{CF}}{\mathrm{CMV} / 365}
\end{array}
$$

Équation 11

Le délai requis pour convertir les stocks en encaisse établit une relation entre le stock de liquidité et la notion de flux monétaires. Richards et Laughlin (1980) définissent le cycle de conversion de l'encaisse (équation 10) comme le délai requis pour convertir en encaisse les stocks (S) et les comptes clients (CC), délai qui est réduit de celui nécessaire pour payer les comptes fournisseurs (CF). L'évaluation en jours s'établit en additionnant le délai de détention des stocks (DDS) au délai de recouvrement des comptes clients (DDCC), ce total étant réduit du délai de paiement des comptes fournisseurs (DPCF). Cette mesure fournit un indicateur de la rapidité de conversion des actifs et passifs circulant en encaisse, tout en précisant l'amplitude du cycle. La mesure est également indicatrice de la qualité de gestion du fonds de roulement. Cette mesure du cycle de conversion de l'encaisse gagne en validité lorsque les ventes sont uniformes et non cycliques. La seconde mesure, proposée par De la Bruslerie (1999), convertit les valeurs calculées en flux monétaires ainsi que sur une base commune, le chiffre d'affaires, corrigeant par des coefficients d'ajustement deux des déficiences relevées par l'auteur à la mesure du cycle de conversion de l'encaisse.

Ces mesures n'informent pas sur la valeur du stock de liquidité ni des flux monétaires futurs et surtout n'indiquent pas à l'utilisateur le montant disponible pour s'acquitter d'un engagement financier.

Les prochaines mesures traitent davantage de l'impact des flux monétaires sur la liquidité.

\subsubsection{Les mesures de flux monétaires}

Ces mesures constituent une réponse à la critique fondamentale émise sur les mesures émanant du bilan qui ne traitent qu'indirectement de l'impact des flux monétaires sur la valeur observée. La plupart d'entre elles proviennent en tout ou en partie de l'état des flux de trésorerie. Quatre mesures de flux monétaires répertoriées dans la littérature sont présentées:

1. L'efficacité de gestion de l'encaisse $(E G E)=\frac{\text { Flux monétaires d'exploitation }}{\text { Ventes }}$

Équation 12 
2. L'indice de liquidité courant $\left(\right.$ ILC) $=\frac{\text { Espèces }+ \text { Quasi-esp. }+ \text { Flux monétaires d'exploitation }}{\text { Passif à court terme }}$

Équation 13

3. La capacité

de remboursement $(\mathrm{CR})=\frac{\text { Bénéfice net }+ \text { Amortissement }+ \text { Intérêts }- \text { Dividendes }}{\text { Capital }+ \text { Intérêts }}$

Équation 14

4. Le lambda $(\lambda)=\frac{\text { Stock de liquidité }+ \text { Flux monétaires anticipés }}{\text { Incertitude sur les flux monétaires }}$

Équation 15

La première de ces mesures décrit la capacité de l'entreprise, en pourcentage des ventes, à fournir des liquidités. On l'obtient en calculant les flux monétaires engendrés par l'exploitation sur les ventes, ces flux monétaires constituant le total des activités d'exploitation à l'état des flux de trésorerie. Bien que cette mesure traite des flux de liquidité, elle a l'inconvénient d'être muette sur les stocks de liquidité. Elle ne procure également pas d'information sur les activités d'investissement et de financement ni sur les variations potentielles de liquidité provenant de la marge de crédit, donc n'indique pas à l'utilisateur le montant disponible pour respecter un engagement financier.

La deuxième mesure, présentée par Fraser (1983), vise, selon l'auteur, à complémenter le ratio de fonds de roulement. C'est une mesure de flux monétaires qui indique la capacité de l'entreprise à respecter ses engagements à court terme. Cette mesure a l'avantage de traiter de notions de stock et de flux de liquidité. Elle est cependant incomplète, car elle ne traite pas des activités d'investissement et de financement ni des fluctuations possibles de la marge de crédit, outre le fait qu'elle évalue davantage la solvabilité (à son dénominateur) que la liquidité.

La troisième mesure est surtout employée par les institutions financières pour déterminer la capacité de remboursement des engagements financiers pris par les PME. Cette mesure omet de traiter des variations dans les actifs induits par la croissance; elle est donc plus pertinente pour les entreprises avec une croissance stable. De plus, elle ne considère pas le stock de liquidité ni les variations induites par la marge de crédit.

La dernière mesure est sans aucun doute la plus exhaustive. Le lambda d'Emery (1984) intègre les notions de stock et de flux de liquidité au numérateur ainsi que des mesures de dispersion au dénominateur. Elle sert donc 
TABLEAU 1

Comparaison entre les mesures de liquidité

\begin{tabular}{|c|c|c|c|c|c|c|c|c|c|}
\hline & RFDR & RLI & CCE & ССЕА & SLN & EGE & ILC & CR & $\Lambda$ \\
\hline $\begin{array}{l}\text { Stock } \\
\text { de liquidité }\end{array}$ & $\mathrm{x}$ & $\mathrm{x}$ & & & $\mathrm{x}$ & & $\mathrm{x}$ & & $\mathrm{x}$ \\
\hline $\begin{array}{l}\text { Espèces et } \\
\text { quasi-espèces }\end{array}$ & & & & & $\mathrm{x}$ & & $\mathrm{x}$ & & $\mathrm{x}$ \\
\hline
\end{tabular}

Solde inutilisé

de la marge

de crédit

Découvert

bancaire

\begin{tabular}{lcccc}
\hline $\begin{array}{l}\text { Flux } \\
\text { monétaires }\end{array}$ & $\mathrm{x}$ & $\mathrm{x}$ & $\mathrm{x}$ & $\mathrm{x}$ \\
\hline $\begin{array}{l}\text { Activités } \\
\text { d'exploitation }\end{array}$ & $\mathrm{x}$ & $\mathrm{x}$ & & $\mathrm{x}$ \\
\hline $\begin{array}{l}\text { Activités } \\
\text { d'investis- } \\
\text { sement }\end{array}$ & & & & $\mathrm{x}$ \\
\hline $\begin{array}{l}\text { Activités } \\
\text { de financement }\end{array}$ & & & \\
\hline
\end{tabular}

à évaluer non seulement les liquidités actuelles, mais également l'influence des flux de liquidité futurs sur la liquidité actuelle. Le calcul donne une estimation de la probabilité de voir la liquidité se tarir lors de la prochaine période.

La principale critique adressée à cette méthode vient du calcul du dénominateur. Lors de l'analyse du lambda, le dénominateur est lié à la volatilité des flux monétaires. Dans le contexte d'une jeune PMEC, peu de données historiques servent à sa mesure; pour une PMEC plus mature, la croissance induit le changement et la complexité. Ainsi, de période en période, la PMEC change et la mesure de la volatilité estimée par l'écart type ou la dispersion est basée sur des valeurs qui ne portent pas sur le même objet. Donc, la mesure serait biaisée du seul fait que la PMEC est en croissance et, dès lors, difficilement utilisable, car imprécise dans le cas des PMEC. De plus, l'indice lambda n'informe pas sur le niveau (ou réservoir) de liquidité disponible pour régler des engagements financiers, mais sur la probabilité de voir ce dernier se tarir. C'est donc une mesure différente de celle développée dans ce texte. 


\subsection{Comparaison des mesures de liquidité}

Le tableau 1 présente une synthèse des mesures recensées et leurs liens avec les constituantes du stock et des flux de liquidité. Il en ressort que le ratio de fonds de roulement, celui le plus utilisé dans la littérature, ne satisfait pas aux exigences d'exhaustivité postulées comme condition nécessaire au développement d'une mesure. En fait, à part le lambda d'Emery, chacune des autres mesures comporte des éléments non couverts, soit dans le stock ou dans les flux de liquidité. Ainsi, sur une base conceptuelle, l'indicateur recherché ne peut être constitué d'aucune de celles-ci.

Les particularités des PMEC incitent également à préconiser une mesure qui sera plus centrée sur le niveau de la liquidité, ainsi que sur les éléments qui affectent ce niveau, ce qui exclut également le lambda comme mesure choisie.

La prochaine section poursuit la réflexion sur le choix de la mesure en testant la validité et la puissance du modèle développé dans le présent article, par rapport aux autres indicateurs recensés dans la littérature.

\section{Méthode de recherche}

Les données utilisées lors de cette recherche proviennent d'une base de données privée développée par le Laboratoire de recherche sur la performance des entreprises (LaRePE) de l'Université du Québec à Trois-Rivières dans le cadre d'activités de diagnostic d'entreprise (St-Pierre et Delisle,2006). Le questionnaire a été développé en collaboration avec des chefs d'entreprise sur une période de 18 mois dans le but de créer un outil de diagnostic sur les différentes dimensions de la performance, incluant les dimensions financières. Un comité de travail mixte, formé de chefs d'entreprise et de chercheurs, a ainsi travaillé étroitement en collaboration et sur une base régulière (au moins une réunion par mois) pour arriver à un questionnaire complet et facilement compréhensible pour les chefs d'entreprise. Le questionnaire a été développé par une équipe de chercheurs en finance, développement organisationnel, économie, gestion des ressources humaines, logistique, génie industriel et système d'information, et a été validé auprès des dirigeants des PME, afin de s'assurer de la pertinence de son contenu et de la clarté de la formulation des questions. Les données proviennent de PME manufacturières québécoises ayant entre 5 et 500 employés. Deux instruments de mesure servent à créer la base de données, soit un questionnaire, ainsi que les états financiers des PME pour les cinq années financières complétées au moment de remplir le questionnaire. 
L'utilisation de données secondaires comporte des limites sur l'applicabilité de l'information aux fins de la recherche. En revanche, ces données sont recueillies auprès d'entreprises manufacturières qui doivent gérer des stocks et des comptes clients, et qui utilisent souvent une marge de crédit bancaire pour combler leurs besoins de liquidités. L'échantillon recueilli constitue un «échantillon non probabiliste volontaire», car les individus fournissaient volontairement leurs données en échange d'un rapport de diagnostic. Pour des fins de validité externe, cet échantillon ne peut être généralisé à la population des PME.

L'échantillon initial comportait 418 entreprises, desquelles nous avons extrait un sous-échantillon d'entreprises «en croissance». La littérature sur les PME en croissance et les «gazelles » fixe à $20 \%$ la croissance annuelle du chiffre d'affaires des PME sur une période allant de trois à cinq ans. Pour établir l'échantillon, la moyenne géométrique de croissance du chiffre d'affaires lors de cinq années a été préférée à la moyenne arithmétique; l'échantillon retenu est plus circonscrit de cette manière. Un total de 55 entreprises répondait à cette contrainte. Toutefois, 6 entreprises ont été rejetées par manque de données, ce qui a réduit l'échantillon de base à 49 entreprises.

Le tableau 2 présente certaines statistiques descriptives de l'échantillon. Un examen des données révèle que la croissance des entreprises est souvent irrégulière et non linéaire, ce qui confirme les résultats de Mustar (2001).

\section{TABLEAU 2}

Statistiques descriptives de l'échantillon

\begin{tabular}{lcccc}
\hline & $\begin{array}{c}\hat{A} g e \\
\text { de la PMEC }\end{array}$ & $\begin{array}{c}\text { Ventes } \\
\text { de la PMEC }\end{array}$ & $\begin{array}{c}\text { Nombre } \\
\text { d'employés }\end{array}$ & $\begin{array}{c}\text { Croissance } \\
\text { sur 5 ans }\end{array}$ \\
\hline Moyenne & 15,24 & $8260536 \$$ & 71,53 & $32,76 \%$ \\
\hline Minimum & 6,00 & $556489 \$$ & 7,00 & $21,20 \%$ \\
\hline Maximum & 54,00 & $54746782 \$$ & 405,00 & $59,24 \%$ \\
\hline Écart type & 8,85 & $9789580 \$$ & 73,95 & $9,19 \%$ \\
\hline
\end{tabular}

Afin de répondre à la question de recherche, nous allons tout d'abord effectuer un premier test, soit une analyse de corrélation entre les variables. Le test de corrélation est effectué entre les mesures et le ratio RSL sur les trois années les plus récentes. Puisque l'échantillon retenu comporte un faible nombre d'entreprises, la répétition du test sur trois années fournit plus d'observations, ce qui permet de conclure à la qualité des résultats obtenus tout en minimisant la perte d'entreprises à la suite du manque de données. En outre, cela donne plus de robustesse à nos conclusions. 
Dans un deuxième temps, nous allons effectuer une régression linéaire simple afin de vérifier quel est le meilleur indicateur «stock» de la liquidité future $\left(\mathrm{RSL}_{t}\right)$ parmi les 10 mesures choisies. Le critère de décision consiste alors à choisir la mesure qui produit le meilleur coefficient $\beta$ et la meilleure statistique $t$, donc le meilleur coefficient de détermination. Le test est à nouveau effectué sur trois années, et les résultats peuvent donner une interprétation à la fois sur la force de la relation et la stabilité de cette dernière.

$\operatorname{RSL}_{t}=\alpha+\beta\left(\right.$ Mesure $\left._{i, t-1}\right)+\varepsilon$

Équation 16

Où: Mesure $_{i}=$ Chaque mesure de liquidité (incluant RSL) de l'année précédente.

Dans un troisième temps, nous allons effectuer un test de régression linéaire multiple où la liquidité future $\left(\mathrm{RSL}_{t}\right)$ est estimée par chaque mesure testée à l'équation 16 ainsi que chaque constituante des flux monétaires établie à l'équation 6. Afin de rendre les informations sur les flux monétaires comparables entre les entreprises, chacun des flux monétaires a été divisé par le chiffre d'affaires, d'où la lettre $\mathrm{V}$ qui complète chaque variable. Les variables NFPA et NFPE ont été regroupées, car les informations ne nous permettaient pas d'établir une différenciation entre les deux sources de recettes.

$$
\begin{aligned}
& \operatorname{RSL}_{t}=\alpha+\beta_{1}\left(\operatorname{Mesure}_{i, t}-1\right)+\beta_{2}\left(\mathrm{FAGV}_{t}\right)-\beta_{3}\left(\mathrm{FDRV}_{t}\right)-\beta_{4}\left(\mathrm{DIVIDV}_{t}\right) \\
& -\beta_{5}\left(\mathrm{PCTDLTV}_{t}\right)-\beta_{6}\left(\mathrm{NIMMV}_{t}\right)+\beta_{7}\left(\mathrm{MCV}_{t}\right)+\beta_{8}\left(\mathrm{NFPV}_{t}\right)+\varepsilon
\end{aligned}
$$

Équation 17

Ces deux tests de régression vont servir à mesurer si l'indicateur développé, en plus d'être le plus pertinent sur le plan conceptuel, est le meilleur estimateur de la liquidité future. Afin de valider les conclusions tirées sur les PMEC, nous allons effectuer les mêmes tests sur des entreprises à plus faible croissance. L'analyse de régression linéaire simple et multiple a été préférée à celle de type panel afin de démontrer la sensibilité de la mesure au stock et aux flux de liquidité périodiques.

\section{Les résultats}

Partant de l'hypothèse que la mesure RSL sert à calculer le réservoir de liquidités futures, le test de corrélation a été effectué en comparant les neuf mesures au RSL, pour chacune des trois années précédant le dépôt du questionnaire. L'examen du tableau 3 montre que les ratios de fonds de roulement (RFDR), de liquidité immédiate (RLI) et l'indice de liquidité de 
Fraser (ILC) sont corrélés positivement avec le niveau de $1 \%$ au ratio RSL, et ce, pour chacune des trois années. Les corrélations sont aussi significatives pour le solde de liquidité nette (SLN) pour chacune des années, mais avec le niveau de $5 \%$ pour deux d'entre elles. Les autres ratios présentent une corrélation faible ou inexistante avec le RSL. Des résultats assez similaires sont constatés chez les entreprises en faible croissance ${ }^{3}$; par contre, c'est le ratio de capacité de remboursement bancaire (CR) qui se substitue au SLN comme mesure significative, ce qui montre que cette mesure semble plus appropriée pour les entreprises à faible croissance. Les résultats avec le lambda ne sont pas significatifs, ni dans ces tests de corrélations ni dans les analyses de régression effectuées ultérieurement. La comparaison sous forme de régression ou de corrélation avec ce ratio perd de son sens puisque la probabilité de suffisance de liquidité du lambda est respectée dès que le ratio excède 3. Aussi, le lambda sera-t-il exclu des analyses futures.

TABLEAU 3

\section{Corrélation de Pearson entre les mesures de liquidité chez les PMEC $(n=49)$}

\begin{tabular}{lccccccccc}
\hline & RFDR & RLI & CCE & CCEA & SLN & EGE & ILC & CR & LAMBDA \\
\hline RSL1 & $0,545^{* * *}$ & $0,655^{* * *}$ & $-0,239$ & $-0,311^{* *}$ & $0,511^{* * *}$ & 0,257 & $0,576^{* * *}$ & 0,188 & 0,122 \\
RSL2 & $0,719^{* * *}$ & $0,764^{* * *}$ & 0,009 & $-0,074$ & $0,330^{* *}$ & 0,095 & $0,619^{* * *}$ & 0,267 & 0,202 \\
RSL3 & $0,608^{* * * *}$ & $0,619^{* * *}$ & 0,001 & 0,091 & $0,347^{* *}$ & $-0,147$ & $0,456^{* * *}$ & 0,254 & 0,208 \\
\hline ** La corrélation est significative au niveau 0,05 (bilatéral). & & & & \\
***La corrélation est significative au niveau 0,01 (bilatéral). & & & & \\
\hline
\end{tabular}

Les premières analyses de régression linéaire simple ont été effectuées sur trois années afin de donner plus de robustesse aux résultats. Un total de 27 régressions a été réalisé, une pour chacune des neuf mesures, à chaque année. Les résultats sont présentés au tableau 4. Les rectangles sous chaque variable indépendante montrent l'essentiel des résultats de la régression effectuée sur chaque variable dépendante apparaissant dans la première colonne. La variable dépendante de l'année $t$ est estimée par l'indicateur «stock» de l'année précédente.

3. Afin de ne pas alourdir le document avec leur présentation exhaustive, les résultats peuvent être fournis par l'auteur sur demande. 


\section{TABLEAU 4}

\section{Régressions linéaires entre les neuf mesures de liquidité et le RSL sur trois ans}

\begin{tabular}{|c|c|c|c|c|c|c|c|c|c|}
\hline $\begin{array}{l}\text { Variable } \\
\text { dépendante }\end{array}$ & $\begin{array}{l}\text { Variab } \\
\text { indépe }\end{array}$ & $\begin{array}{l}\text { le } \\
\text { ndante }\end{array}$ & & & & & & & \\
\hline & RSL2 & RFDR2 & RLI2 & CCE2 & CCEA2 & SLN2 & EGE2 & ILC2 & CR2 \\
\hline RSL1Bêta & 0,623 & 0,441 & 0,598 & $-0,220$ & $-0,271$ & 0,434 & 0,068 & 0,523 & 0,244 \\
\hline$t$ & 5,458 & 3,367 & 5,119 & $-1,543$ & $-1,930$ & 3,303 & 0,464 & 4,203 & 1,725 \\
\hline Signification & 0,000 & 0,002 & 0,000 & 0,130 & 0,060 & 0,002 & 0,645 & 0,000 & 0,091 \\
\hline $\mathrm{R}^{2}$ ajusté & 0,375 & 0,177 & 0,344 & 0,028 & 0,054 & 0,171 & $-0,017$ & 0,258 & 0,039 \\
\hline \multirow[t]{2}{*}{ Statistique F } & 29,78 & 11,335 & 26,200 & 2,300 & 3,723 & 10,909 & 0,196 & 17,666 & 2,974 \\
\hline & RSL3 & RFDR3 & RLI3 & CCE3 & CCEA3 & SLN3 & EGE3 & ILC3 & CR3 \\
\hline RSL2 Bêta & 0,441 & 0,405 & 0,322 & 0,071 & $-0,011$ & 0,188 & 0,232 & 0,258 & 0,109 \\
\hline$t$ & 3,367 & 3,038 & 2,335 & 0,490 & $-0,075$ & 1,316 & 1,635 & 1,834 & 0,750 \\
\hline Signification & 0,002 & 0,004 & 0,024 & 0,626 & 0,941 & 0,195 & 0,109 & 0,073 & 0,457 \\
\hline $\mathrm{R}^{2}$ ajusté & 0,177 & 0,146 & 0,085 & $-0,016$ & $-0,021$ & 0,015 & 0,034 & 0,047 & $-0,009$ \\
\hline \multirow[t]{2}{*}{ Statistique $\mathrm{F}$} & 11,336 & 9,231 & 5,451 & 0,240 & 0,006 & 1,731 & 2,720 & 3,363 & 0,562 \\
\hline & RSL4 & RFDR4 & RLI4 & CCE4 & CCEA4 & SLN4 & EGE4 & ILC4 & CR4 \\
\hline RSL3 Bêta & 0,665 & 0,479 & 0,513 & 0,272 & 0,205 & 0,220 & 0,311 & 0,452 & 0,349 \\
\hline$t$ & 5,941 & 3,738 & 4,099 & 1,936 & 1,435 & 1,545 & 2,246 & 3,470 & 2,551 \\
\hline Signification & 0,000 & 0,001 & 0,000 & 0,059 & 0,158 & 0,129 & 0,029 & 0,001 & 0,014 \\
\hline $\mathrm{R}^{2}$ ajusté & 0,417 & 0,213 & 0,248 & 0,054 & 0,022 & 0,028 & 0,078 & 0,187 & 0,103 \\
\hline Statistique $\mathrm{F}$ & 35,301 & 13,976 & 16,799 & 3,748 & 2,059 & 2,387 & 5,027 & 12,043 & 6,509 \\
\hline
\end{tabular}

Les résultats indiquent que c'est le ratio RSL qui affiche la relation la plus significative avec la liquidité future, grâce à un coefficient de détermination ajusté et une statistique $\mathrm{F}$ supérieurs à ceux de toute autre mesure, confirmant sa capacité prédictive de la liquidité future d'une PMEC. C'est aussi l'indicateur qui présente les coefficients de régression les plus élevés. Le $\mathrm{R}^{2}$ ajusté varie entre $17,7 \%$ et $41,7 \%$, ce qui montre que le stock de liquidité de l'année précédente n'explique qu'une partie du stock de liquidité de l'année courante et que le pouvoir de prévision de la mesure fluctue au fil des années. 
Afin d'améliorer le niveau de précision des tests, nous avons inclus les variables de flux monétaires dans les analyses de régression. La deuxième série d'analyses de régression exprime le lien entre les variables «stocks» de la période précédente et celles des flux monétaires de la période actuelle. Étant donné que ces éléments expliquent en grande partie le niveau de liquidité attendu, les coefficients de détermination attendus avec ces régressions linéaires multiples devraient surpasser ceux obtenus avec les régressions linéaires simples. Les régressions ont été effectuées sur l'intervalle temporel de trois ans. Encore ici, le ratio RSL s'est mieux comporté que l'ensemble des autres mesures ${ }^{4}$.

Le tableau 5 indique les résultats des analyses de régression linéaire multiple lorsque l'indicateur «stock» de liquidité était le ratio RSL de la période précédente. Sur les trois années observées, les coefficients de détermination obtenus oscillent entre 47,2\% et 74,6\%.L'ajout des flux monétaires tels les fonds autogénérés sur les ventes et le fonds de roulement sur les ventes augmente à chaque période le degré de signification de la régression. Lors de deux des trois années, la variable mesurant l'apport en immobilisations a un impact très significatif sur la liquidité. Ces résultats indiquent l'importance de la variation des flux monétaires annuels dans la prise en compte du stock de liquidité.

Des tests effectués sur des entreprises à plus faible croissance confirment la pertinence d'estimer les flux monétaires dans l'évaluation de la liquidité future chez les entreprises en croissance. La hausse du coefficient de détermination lorsque les flux de liquidité des PMEC sont ajoutés au stock de liquidité est plus élevée chez ces dernières que chez celles dont la croissance est plus stable. D'autres tests montrent également une absence de corrélation entre les flux monétaires de deux périodes consécutives. Ainsi, les flux monétaires passés sont de mauvais indicateurs des flux monétaires futurs chez les entreprises en croissance de notre échantillon. Ces résultats renforcent la pertinence de l'analyse de la liquidité future chez les PMEC, liquidité estimée par le stock de liquidité actuel et les flux monétaires futurs.

Il appert de ces résultats que:

- Le stock de liquidité constitue la variable la plus significative dans la détermination du niveau de liquidité future.

- Les fonds autogénérés contribuent à la hausse du niveau de liquidité, ce qui est conforme à la théorie de l'ordre hiérarchique; la hausse de la marge de crédit et les fonds propres externes suivent dans l'ordre.

4. Encore une fois, afin de ne pas alourdir le document, les résultats peuvent être fournis par l'auteur sur demande. 


\section{TABLEAU 5}

Régressions multiples mettant en relation le RSL et les composantes des flux monétaires à la liquidité future des PMEC

\begin{tabular}{|c|c|c|c|c|c|c|c|c|c|}
\hline $\begin{array}{l}\text { Variable } \\
\text { dépendante }\end{array}$ & $\begin{array}{l}\text { Variab } \\
\text { indépe }\end{array}$ & le & & & & & & & \\
\hline & & FAGV1 & FDRV1 & DIVIDV1 & NFPV1 & NIMMV1 & MCV1 & CAPV1 & RSL2 \\
\hline RSL1 Bêta & & 0,547 & $-0,303$ & $-0,074$ & 0,241 & $-0,120$ & 0,066 & $-0,164$ & 0,569 \\
\hline$t$ & & 3,443 & $-2,546$ & $-0,617$ & 1,598 & $-1,015$ & 0,563 & $-1,173$ & 5,073 \\
\hline VIF & & 2,293 & 1,288 & 1,313 & 2,07 & 1,267 & 1,265 & 1,776 & 1,141 \\
\hline $\mathrm{R}^{2}$ ajusté & 0,472 & & & & & & & & \\
\hline Statistique F & 6,357 & & & & & & & & \\
\hline Sig & 0,000 & 0,001 & 0,015 & 0,541 & 0,118 & 0,316 & 0,577 & 0,248 & 0,000 \\
\hline
\end{tabular}

FAGV2 FDRV2 DIVIDV2 NFPV2 NIMMV2 MCV2 CAPV2 RSL3

\begin{tabular}{|c|c|c|c|c|c|c|c|c|c|}
\hline RSL2 Bêta & & 0,737 & $-0,423$ & $-0,234$ & 0,176 & $-0,744$ & 0,149 & $-0,245$ & 0,579 \\
\hline$t$ & & 6,397 & $-4,763$ & $-2,498$ & 2,111 & $-7,022$ & 1,730 & $-2,725$ & 6,738 \\
\hline VIF & & 2,016 & 1,197 & 1,329 & 1,062 & 1,681 & 1,128 & 1,226 & 1,122 \\
\hline $\mathrm{R}^{2}$ ajusté & 0,684 & & & & & & & & \\
\hline Statistique & 14,006 & & & & & & & & \\
\hline Sig & 0,000 & 0,000 & 0,000 & 0,017 & 0,041 & 0,000 & 0,091 & 0,009 & 0,000 \\
\hline & & FAGV3 & FDRV3 & DIVIDV3 & NFPV3 & NIMMV3 & MCV3 & CAPV3 & 3 RSL4 \\
\hline RSL3 Bêta & & 0,58 & $-0,555$ & $-0,089$ & 0,077 & $-0,438$ & 0,154 & $-0,167$ & 0,688 \\
\hline$t$ & & 5,347 & $-5,637$ & $-1,165$ & 0,951 & $-3,988$ & 2,046 & $-2,01$ & 8,969 \\
\hline VIF & & 2,226 & 1,832 & 1,102 & 1,255 & 2,281 & 1,071 & 1,312 & 1,113 \\
\hline $\mathrm{R}^{2}$ ajusté & 0,746 & & & & & & & & \\
\hline Statistique & 18,624 & & & & & & & & \\
\hline Sig & 0,000 & 0,000 & 0,000 & 0,251 & 0,348 & 0,000 & 0,047 & 0,051 & 0,000 \\
\hline
\end{tabular}


- Les besoins en éléments d'actif à court et à long terme influencent significativement les liquidités des PMEC.

- Les propriétaires-dirigeants restreignent les prélèvements de dividendes lors de la croissance.

- Les versements de capital sur la dette à long terme exigibles en moins d'un an influencent significativement le niveau de liquidité des PMEC.

Les résultats des analyses confirment également le choix de la mesure RSL comme meilleur indicateur de la liquidité des PME, si on la compare aux autres mesures répertoriées dans la littérature. Ce choix renforce les constatations émises dans le cadre conceptuel.

\section{Conclusion}

L'objectif de cet article consistait à développer une mesure exhaustive de liquidité pour les PMEC. Le développement conceptuel et empirique de l'article montre que les mesures traditionnelles ne répondent pas à cet objectif. De surcroît, elles ne permettent pas de connaître le montant disponible pour régler un arrangement financier.

Il a été montré théoriquement et empiriquement que le ratio RSL constitue la meilleure mesure pour établir le niveau de liquidité des PMEC. Elle constitue une mesure performante d'estimation des besoins financiers des PMEC. Il s'agit en outre d'une mesure concrète, facile à calculer et à interpréter, qui est sensible aux prévisions du chiffre d'affaires.

L'échantillon volontaire et non aléatoire constitue la principale limite de cette recherche, les résultats ne pouvant être généralisés à une population d'entreprises manufacturières. De plus, le recours à des données secondaires limite l'utilisation désirée de l'information financière obtenue.

Les principales avenues de recherche touchent la confirmation de la validité de la mesure par l'application à d'autres échantillons. Le développement en entreprise en vue d'une application concrète constitue également une avenue de recherche privilégiée. 


\section{Bibliographie}

BECCHETTI, L. et G. TROVATO (2002), «The determinants of growth for small and medium sized firms. The role of the availability of external finance», Small Business Economics, vol. 19, nº 4, p. 291.

BERGER, A.N. et G.F. UDELL (1995), «Relationship between lending and lines of credit in small firm finance», The Journal of Business, vol. 68, $\mathrm{n}^{\circ} 3$, p. 351-381.

BERGER, A.N. et G.F. UDELL (1998), «The economics of small business finance: the roles of private equity and debt markets in the financial growth cycle», Journal of Banking and Finance, vol. 22, p. 613-673.

BINKS, M.R. et C.T. ENNEW (1996), «Growing firms and the credit constraint», Small Business Economics, vol. 8, n 1, p. 17-30.

BIRD, R.G. et D.P.J. JÜTTNER (1975), «The financing of small business in the manufacturing sector», Research paper No. 69, School of Economic and Financial Studies, Macquarie University, Sidney.

BIRLEY, S. et P. WESTHEAD (1990), «Growth and performance contrasts between "types" of small firms », Strategic Management Journal, vol. 11, p. 535-537.

CARPENTER, R.E. et B.C.PETERSEN (2002), «Is the growth of small firms constrained by internal finance?», The Review of Economics and Statistics, vol. 84, $\mathrm{n}^{\circ}$ 2, p. 298-309.

CHURCHILL, N.C. et V.L. LEWIS (1983), «The five stages of small business growth», Harvard Business Review, vol. 61, n 3, p. 38-50.

COOLEY, P.L. et C.E. EdWARDS (1983), «Financial objectives of small firms», American Journal of Small Business, vol. 8, $\mathrm{n}^{\circ}$ 1, p. 27-33.

DE LA BRUslerie, H. (1999), Analyse financière et risque de crédit, Paris, Dunod, $398 \mathrm{p}$.

DELMAR, F., P. DAVIDSSON et W.B. GARTNER (2003), «Arriving at the high-growth firm », Journal of Business Venturing, vol. 8, n 2, p. 189-216.

EMERY, G.W. (1984), «Measuring short-term liquidity», Journal of Cash Management, juillet-août, p. 25-32.

FRASER, L. (1983), «Cash flow from operations and liquidity analysis : a new financial ratio for commercial lending decisions », The Journal of Commercial Bank Lending, vol. 66, $\mathrm{n}^{\circ} 3$, p. 45-52.

HOLMES, S. et P. KENT (1991), «An empirical analysis of the financial structure of small and large Australian manufacturing enterprises », Journal of Small Business Finance, vol. 1, n 2, p. 141-154.

HOWORTH, C.A. (2001), «Small firms' demand for finance: a research note», International Small Business Journal, vol. 19, n 4, p. 78-88.

HYYTINEN, A. et O. TOIVANEN (2005), «Do financial constraints hold back innovation and growth? Evidence on the role of public policy», Research Policy, vol. 34, p. 1385-1403. 
JENSEN, M.C. et W. MECKLING (1976), «Theory of the firm: managerial behaviour, agency costs and capital structure», Journal of Financial Economics, vol. 3, p. 305-360.

JULIEN, P.-A. (2002), Les PME à forte croissance. L'exemple de 17 gazelles dans 8 régions du Québec, Québec, Presses de l'Université du Québec, 243 p.

LECORnU, M.R., R.G.P. MCMAHON, D.M. ForSAith et A.M.J. STANGER (1996), «The small enterprise financial objective function », Journal of Small Business Management, vol. 34, n 3, p. 1-15.

LEMKE, K.W. (1970), «The evaluation of liquidity: an analytical study», Journal of Accounting Research, vol. 8, $\mathrm{n}^{\circ}$ 1, p. 47-77.

MCMAHON, R.G.P. (2001), «Growth and performance of manufacturing SMEs: the influence of financial management characteristics », International Small Business Journal, vol. 19, n 3, p. 10.

MCMAHON, R.G.P. (2004), «Financial slack amongst manufacturing SMEs from Australia's business longitudinal survey: an explanoratory study», Flinders University, Commerce Research Paper Series, vol. 4, nº 7, 40 p.

MUSTAR, P. (2001), «Diversité et unité des entreprises à forte croissance du secteur manufacturier en France», Revue internationale PME, vol. 14, nos 3-4, p. 67-89.

MYERS, S.C. (1984), «The capital structure puzzle», The Journal of Finance, vol. 39, $\mathrm{n}^{\circ} 3$, p. $575-572$.

MYERS, S.C. et N.S. MAJLUF (1984), «Corporate financing and investment decisions when firms have information that investors do not have», Journal of Financial Economics, vol. 13, no 2, p. 187-222.

NAFFZIGER, D.W., J.S. HORNSBY et D.F. KURATKO (1994), «A proposed research model of entrepreneurial motivation », Entrepreneurship Theory and Practice, vol. $18, n^{\circ} 3$, p. 29-43.

RAY, G. et P. HUTCHINSON (1983), The Financing and Financial Control of Small Enterprise Development, Aldershot, Gower.

RICHARDS, V.D. et E.J. LAUGHLIN (1980), «A cash conversion cycle to liquidity analysis », Financial Management, vol. 9, n 1, p. 32-38.

ST-PIERRE, J. (1999), La gestion financière des PME. Théories et pratiques, Québec, Presses de l'Université du Québec, 322 p.

St-PIERRE, J., R. BEAUDOIN et M. DESMARAIS (2002), Le financement des PME canadiennes: satisfaction, accès, connaissance et besoins, Industrie Canada, 129 p.

ST-PIERRE, J. et S. DELISLE (2006), «An expert diagnosis system for the benchmarking of SMEs' performance», Benchmarking: An International Journal, vol. 13, $\mathrm{n}^{\text {os }} 1-2$, p. 106-119.

United States Government PRINTING OFFICE (2007), The Small Business Economy for Data Year 2006: A Report to the President, 368 p. 
VAN AUKen, H. et L. NEELEY (1996), «Evidence of bootstrap financing among small start-up firms », Entrepreneurial and Small Business Finance, vol. 5, nº 3, p. 235-249.

WALKER, E. et A. BROWN (2004), «What success factors are important to small business owners? », International Small Business Journal, vol. 22, nº 6, p. 577-594.

WELSH, J.A. et J.F. WHITE (1978), «Return on investment... or liquidity? A manager's dilemma », Journal of Small Business Management, vol. 16, nº 2, p. 14-22.

WELSH, J.A. et J.F. WHITE (1981), «A small business is not a little big business », Harvard Business Review, vol. 59, nº 4, p. 18-29.

WINBORG, J. et H. LANDSTRÖM (2001), «Financial bootstrapping in small businesses : examining small business managers' resource acquisition behaviors », Journal of Business Venturing, vol. 16, n 3, p. 235-254.

ZOPPA,A. et R.G.P.MCMAHON (2002), «Pecking order theory and the financial structure of manufacturing SMEs from Australia's business longitudinal survey», Flinders University, Commerce Research Paper Series, vol. 2, nº 1, 29 p. 